\title{
Subthreshold Mismatch in Nanometer CMOS at Cryogenic Temperatures
}

\author{
P. A. 'T HART ${ }^{\circledR 1}$, M. BABAIE ${ }^{\circledR}$ (Member, IEEE), EDOARDO CHARBON $^{\circledR}$ (Fellow, IEEE), \\ ANDREI VLADIMIRESCU1,3,4, AND FABIO SEBASTIANO 1 (Senior Member, IEEE) \\ 1 AQUA, TU Delft, 2628 CD Delft, The Netherlands \\ 2 Department of Microengineering, École Polytechnique Fédérale de Lausanne, 2002 Lausanne, Switzerland \\ 3 ISEP, 75006 Paris, France \\ 4 Berkeley Wireless Research Center, UC Berkeley, Berkeley, CA 94704, USA \\ CORRESPONDING AUTHOR: P. A. 't HART (e-mail: p.a.thart@tudelft.nl) \\ This work was supported by Intel.
}

(Invited Paper)

\begin{abstract}
Cryogenic device models are essential for the reliable design of the cryo-CMOS electronic interface necessary to build future large-scale quantum computers. This paper reports the characterization of the drain-current mismatch of NMOS and PMOS devices fabricated in a commercial 40-nm bulk CMOS process over the temperature range from $4.2 \mathrm{~K}$ to $300 \mathrm{~K}$. By analysing the variability of device parameters over a wide range of device area and length, the validity of the Pelgrom area-scaling law is assessed for the threshold voltage, the current factor and the subthreshold swing. The Croon model is employed to model the drain-current mismatch in moderate to strong inversion, while the weak inversion region is modeled by taking the subthreshold slope variability into account. This results in the first model capable of predicting CMOS-device mismatch over all operating regions and in the whole temperature range from $300 \mathrm{~K}$ down to $4.2 \mathrm{~K}$.
\end{abstract}

\section{INDEX TERMS}

\section{INTRODUCTION}

Quantum computers promise to solve several problems that are beyond the capability of today's supercomputers. They operate by processing the information stored in quantum bits (qubits), which must be typically cooled to deep-cryogenic temperatures below $1 \mathrm{~K}$ for proper operation and therefore reside in dilution refrigerators [1]. Although the actual computations are carried out by qubits, classical electronics is still required to control and readout the state of the qubits. Current state-of-the-art quantum processors comprise only a few tens of qubits [2], which are directly wired to conventional room-temperature control electronics. However, future quantum computers will require thousands or even millions of qubits in order to address any practical problem. Scaling to such a large number of qubits will be unpractical when using the current approach, as it will become infeasible to wire the thousands of cables required to connect the cryogenic qubits to the room-temperature electronics. To overcome these constraints, the control electronics can be placed in close proximity to the qubits, but, this requires that the classical electronics also operate at cryogenic temperatures. Since typical dilution refrigerators offer very low-cooling power $(<1 \mathrm{~mW})$ at the mK-temperature stage, the preferred operating temperature for the electronics is above $4.2 \mathrm{~K}$, at which significant cooling power ( $>1 \mathrm{~W}$ ) can be provided [3].

Nanometer CMOS operating at cryogenic temperature (cryo-CMOS) is the technology of choice by virtue of its high speed/bandwidth capability, its high level of integration and the potential to operate at temperatures as low as $30 \mathrm{mK}$ [4], [5], which together enable the complex circuits required to control a very large number of qubits. Due to the limited cooling power, the power efficiency of those circuits is paramount. A very attractive solution is to operate cryoCMOS circuits in the subthreshold region to minimize power consumption. The availability of accurate device models validated at cryogenic temperatures is indispensable for the 
design of such cryo-CMOS circuits. Although recent work has focused on cryogenic DC and RF models in advanced technology nodes [4], [6]-[8], mismatch models are still lacking for this target temperature range.

Device mismatch can severely limit the performance of many sensitive circuits, such as current/voltage references and data converters, that are essential components of the target cryo-CMOS controller. There is evidence in literature indicating a deterioration of mismatch at low temperatures [9]-[11], thus stressing even more the importance of the cryogenic modeling of this effect. Mismatch has been studied extensively over the military temperature range $\left(-55-125^{\circ} \mathrm{C}\right)[9]-[13]$, including the subthreshold region [14]-[20]. Work on cryogenic device mismatch, however, has been limited to moderate to strong inversion [21]-[24]. To fill this gap, subthreshold device mismatch of a commercial 40-nm bulk CMOS technology was studied in [25], of which this paper is an extension. In this paper, the behaviour of the subthreshold drain current $\left(I_{D}\right)$ is studied as a function of device geometry over the temperature range from $300 \mathrm{~K}$ (room temperature; RT) down to $4.2 \mathrm{~K}$ (liquid Helium temperature; LHT). In addition to the variability of threshold voltage $\left(V_{T H}\right)$ and current factor $(\beta)$, the subthreshold swing $(S S)$ variability is specifically investigated for its impact on the subthreshold mismatch. Moreover, the validity of the Pelgrom area-scaling law for these three parameters is also assessed at both room and cryogenic temperatures. Furthermore, this work demonstrates a complete model of the drain-current mismatch, valid from the subthreshold to the strong inversion regime over the full temperature range from $300 \mathrm{~K}$ down to $4.2 \mathrm{~K}$, which is obtained by combining the Croon model [26] and the subthreshold mismatch model [17].

This paper is structured as follows: a description of the devices under test, the measurement setup and parameter extraction is given in Section II; the mathematical foundations of the subthreshold and Croon models are established in Section III; the measurement results are presented in Section IV, which are discussed in Section V. Finally, a conclusion is drawn in Section VI.

\section{MEASUREMENT SETUP AND METHODS \\ A. MEASUREMENT SETUP}

Two separate test chips were designed specifically to characterize subthreshold mismatch at cryogenic temperatures. Both chips were fabricated in the same 1.1-V 40-nm bulk CMOS process but in two different batches and comprise thin-oxide NMOS and PMOS devices with standard threshold voltage.

The first test chip (chip \#1) enabled the characterization of three different device geometries laid out in a matched-pair configuration as indicated in Fig. 1a and Fig. 2. The results of the characterization of chip \#1 have also been presented in [25]. A second test chip (chip \#2, Fig. 1b) was designed to include 6 additional device geometries, as indicated in

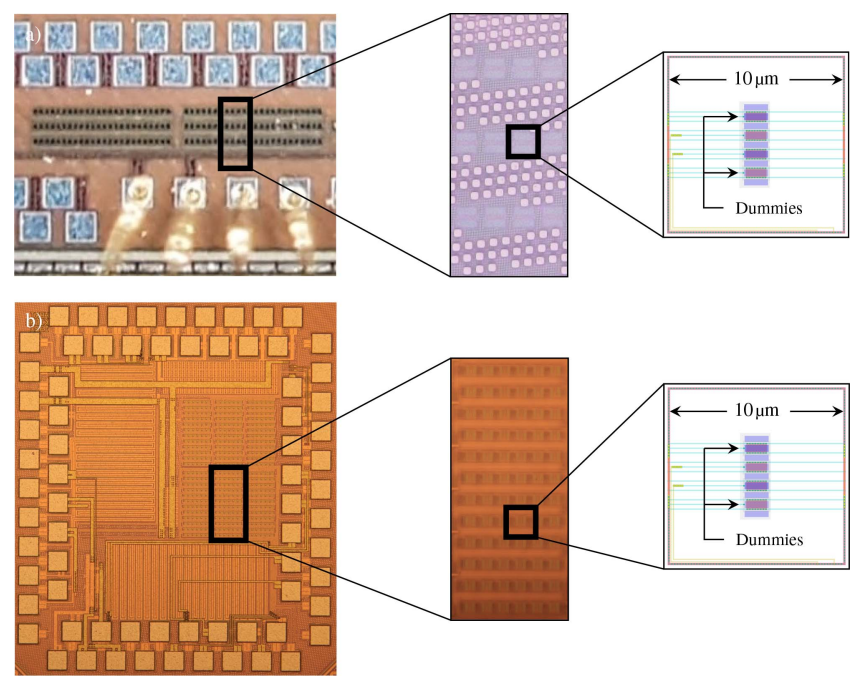

FIGURE 1. Micrograph of the chips used for mismatch characterization: (a) chip \# 1 comprising 3 different device geometries (results also presented in [25]); (b) chip \#2 comprising an extended set of device geometries. Inset shows the layout details of the matched pairs.

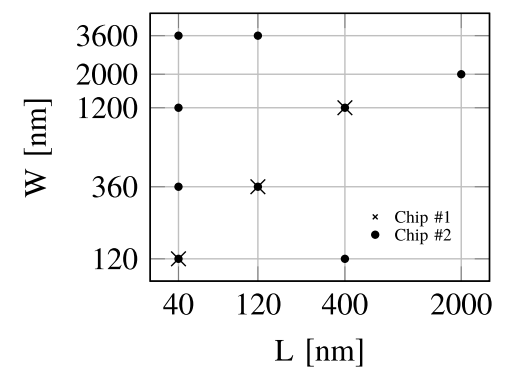

FIGURE 2. Device geometries available in the two test chips.

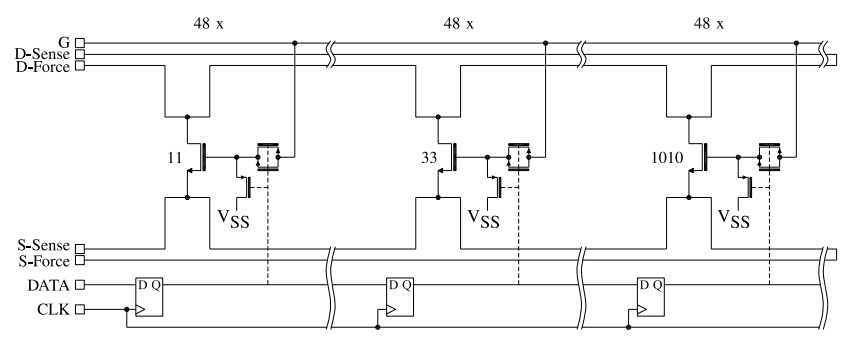

FIGURE 3. Simplified schematic of the NMOS mismatch characterization array of chip \# 1.

Fig. 2, thereby extending the scope of the work, e.g., to include mismatch dependency on device length.

A simplified circuit diagram of the NMOS array is shown in Fig. 3. A similar setup is adopted for PMOS devices. All devices (48 device pairs for each of the 3 geometries) share their drain and source terminals. When a device is selected, its gate is connected to a common bond pad through a transmission gate. When not selected, the gate is either connected to $\mathrm{V}_{\mathrm{SS}}$ (for the NMOS) or $\mathrm{V}_{\mathrm{DD}}$ (for the PMOS). Due to the higher threshold voltage at $4.2 \mathrm{~K}$, the resistance of the transmission gate can significantly increase when biasing the device under test (DUT) in subthreshold. This, combined 
with the gate current of the DUT, caused the anomalous drain current behavior reported in [25]. To overcome this limitation, the supply of the transmission gates was raised by $400 \mathrm{mV}$ (up to $1.5 \mathrm{~V}$ ) for all the characterization at $4.2 \mathrm{~K}$, preventing the anomalous behavior. The transmission gates are controlled by a latched shift register to enable automatic characterization. The potential of the $\mathrm{N}$-well and of the substrate is fixed by contact rings biased at $\mathrm{V}_{\mathrm{DD}}=1.1 \mathrm{~V}$ and $\mathrm{V}_{\mathrm{SS}}=0 \mathrm{~V}$, respectively. Kelvin connections are made to the source and drain to mitigate the effect of series resistance in the measurement path, which is particularly important for the reliable extraction of device parameters in strong inversion where a non-negligible current is expected.

Chip \#1 comprises one NMOS array and one PMOS array, each comprising 3 sets of 24 device pairs. Between the arrays, the shift register is daisy-chained and the gate pad is shared. Source and drain are routed to separate bond pads for each array, in order to minimize leakage. Chip \#2 contains 3 NMOS arrays and 3 PMOS arrays. Their layout and connections are similar to those of chip \#1. Device geometries with similar expected leakage were grouped in each array to increase the measurable subthreshold-current range.

Care was taken to reduce the impact of systematic mismatch, edge effects and mechanical stress by placing the devices in each pair at minimum distance with symmetrical connections, by surrounding them with identical dummy devices and by keeping metallization further than $5 \mu \mathrm{m}$ away from the active devices, as indicated in Fig. 1.

It should be noted that these chips have been fabricated in a different 40-nm bulk CMOS process than the one presented in [21], [22] and that the test structures have been redesigned. This was necessary to minimize leakage and consequently, enable accurate subthreshold-current measurement.

For the electrical characterization, two Keithley 2636B Source Measurement Units (SMUs) were connected to the samples by low-leakage triaxial guarded connections. The measurement setup did not limit the lowest measurable current of the instruments, which is in the range of $10 \mathrm{fA}$. Since the time required to characterize a single die exceeds 36 hours due to the long instrument settling times associated with the low current range, an automated setup with samples directly submerged into liquid Helium is preferred over a manual setup with a cryogenic probestation. Intermediate temperatures between LHT and RT were reached by positioning the sample at different heights in the Helium vapour inside an Helium dewar.

For chip \#1 and chip \#2 a total of six dies (3 dies for chip \#1; 3 dies for chip \#2; 72 matched pairs per geometry) were characterized, both in triode $\left(\left|V_{D S}\right|=50 \mathrm{mV}\right)$ and in the saturation region $\left(\left|V_{D S}\right|=1.1 \mathrm{~V}\right)$ at RT and at LHT. In addition to these two temperatures, the devices on chip \#2 were also measured at $100 \mathrm{~K}$ and a single device of each geometry was characterized at 9 temperature points from $300 \mathrm{~K}$ down to $4.2 \mathrm{~K}$.

All devices have been characterized sequentially in a 3-step procedure: 1) a single device is selected by loading

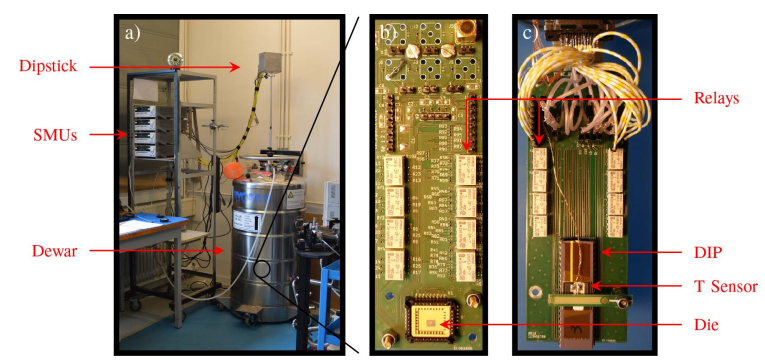

FIGURE 4. Measurement setup. (a): dipstick and instruments; (b) detail of the PCB designed for characterizing chip \# 1; (c) detail of the PCB for characterizing chip \#2.

the correct bit pattern into the digital interface; the drain current is measured as a function of gate-source voltage $\left(V_{G S}\right)$ and recorded; 2) the first measurement is repeated, but with fewer $V_{G S}$ bias points to save time; 3 ) all devices are deselected and the measurement of the chip output is carried out a third time. Step 2 is employed to assess the Short Time Repeatability (STR) of the measurement setup, which is an indication of the reliability of the experiment and is used to guard against measurement error due to bad contacts, sudden (unintended) temperature changes and interference. The STR was always below $0.6 \%$ over the bias range, thus not significantly impacting the measurement results. Step 3 characterizes the inevitable leakage of the array $(\sim 4 \mathrm{nA}$ at RT; <10fA at LHT). To extend the subthreshold range to lower currents, the leakage is subtracted from the value of $I_{D}$ measured in step 1. This corrected data is then used for data analysis.

Since the leakage is weakly bias and temperature dependent, a residual error remains after compensation. Data were discarded for low current ranges, to ensure that this error is always below $10 \%$. The array data was validated against individual devices directly accessible via reserved bond pads in chip \#1.

As shown in Fig. 4, the dies were either wire bonded to a CLCC (chip \#1) or a DIP package (chip \#2). These PCBs were mounted at the tip of a dipstick, which was inserted into a liquid Helium dewar to reach cryogenic temperatures. The die temperature was continuously monitored with a Cernox temperature sensor. Temperature drift between the characterization of paired devices was below $10 \mathrm{mK}$ at LHT, $1.1 \mathrm{~K}$ at RT and $0.3 \mathrm{~K}$ at $100 \mathrm{~K}$. Due to a design error, the PMOS with $\mathrm{W} / \mathrm{L}=120 \mathrm{n} / 40 \mathrm{n}$ suffered from excess leakage current and are not included in the following analysis of subthreshold mismatch.

\section{B. PARAMETER EXTRACTION}

The device parameters relevant for drain-current mismatch modeling in strong inversion, i.e., $V_{T H}$ and $\beta$, were extracted from the $I_{D}-V_{G}$ curves with the use of the Extrapolation in Linear Region (ELR) method [27]. The ELR method was chosen for its reliability over the other two well-known methods, i.e., the constant current [27] and the 3-point [28] method, as it does not depend on arbitrarily chosen extraction 
points. Moreover, it was verified that the ELR results fit very well with the mismatch models employed in this work.

The subthreshold swing is an important device parameter in the subthreshold mismatch model. It is extracted by calculating the inverse slope of a line through two $I_{D}$ points in the subthreshold regime:

$$
S S^{-1}=\frac{\left|\log \left(I_{D_{h i}} / I_{D_{l o}}\right)\right|}{V_{G S_{h i}}-V_{G S_{l o}}},
$$

where the ' $h i$ ' and ' $l o$ ' subscripts indicate a bias point at the edge between weak and moderate inversion and a bias point at the lowest current above the measurement limit, respectively.

As Drain Induced Barrier Lowering (DIBL) decreases the $V_{T H}$ for devices in saturation, the value extracted by ELR in triode will be over-estimated. Therefore, for these devices, the $V_{T H}$ term in Eq. (5) is extracted with the use of the Extrapolation in Saturation Region (ESR) method [27], which captures the impact of DIBL.

\section{MODELING}

The subthreshold drain current is modeled by the simplified exponential equation:

$$
I_{D}=I_{0} e^{\left(V_{G S}-V_{T H}\right) / S S},
$$

where $I_{0}$ is a temperature and geometry-dependent constant. Due to both the exponential nature and the increased $I_{D}$ variability in this operating regime at cryogenic temperatures, Taylor expansion of Eq. (2) becomes impractical, thus making it more appropriate to use a logarithmic transform with base 10 [14], [17]:

$$
\log \left(I_{D}\right) \propto \frac{1}{\ln (10)} \frac{V_{G S}-V_{T H}}{S S} .
$$

If we assume $V_{T H}$ and $S S$ to be the sources of variability, a first-order Taylor expansion of Eq. (3) yields:

$$
\Delta \log \left(I_{D}\right)=\frac{1}{\ln (10)}\left[-\frac{1}{S S} \Delta V_{T H}-\frac{\left(V_{G S}-V_{T H}\right)}{S S} \frac{\Delta S S}{S S}\right] .
$$

The subthreshold drain-current mismatch can then be modeled by the variance of Eq. (4) as [17]:

$$
\begin{aligned}
\sigma_{\Delta \log I_{D}}^{2}=\frac{1}{\ln (10)^{2}}[ & \left(\frac{\sigma_{\Delta V_{T H}}}{\overline{S S}}\right)^{2}+\left(\frac{V_{G S}-V_{T H}}{\overline{S S}} \frac{\sigma_{\Delta S S}}{\overline{S S}}\right)^{2} \\
& \left.+2 \frac{\left(V_{G S}-V_{T H}\right)}{\overline{S S}^{3}} \sigma_{\Delta V_{T H}} \sigma_{\Delta S S} \rho_{\Delta V_{T H}, \Delta S S}\right] .
\end{aligned}
$$

Here the overline indicates the average value over all matched pairs with a given geometry and $\rho_{\Delta V_{T H}, \Delta S S}$ is the correlation coefficient between $\Delta V_{T H}$ and $\Delta S S$. This correlation was found to be statistically insignificant at RT, $100 \mathrm{~K}$ and LHT. Therefore, this term is ignored in the following analysis.

The drain-current mismatch over all temperatures in moderate to strong inversion is modeled by the Croon model [26], as such model has already been proven to apply at cryogenic temperatures in [22]. It can be shown that:

$$
\sigma_{\Delta I_{D} / I_{D}}^{2}=\ln (10)^{2} \sigma_{\Delta \log I_{D}}^{2},
$$

therefore the Croon model is expressed as:

$$
\sigma_{\Delta \log I_{D}}^{2}=\frac{1}{\ln (10)^{2}}\left[\sigma_{\Delta \beta / \beta}^{2}+\left(\frac{\bar{g}_{m}}{\bar{I}_{D}}\right)^{2} \sigma_{\Delta V_{T H}}^{2}\right],
$$

where $g_{m}$ is the transconductance of the devices. For the Croon model in Eq. (7), no correlation term is introduced, as it is typically neglected in moderate/strong inversion [22], [26].

The increased subthreshold mismatch resulting from the increased $S S$ variability cannot adequately be modeled by Eq. (7), therefore the Croon model is only used outside the subthreshold region. In weak inversion, the subthreshold model of Eq. (5) is employed.

Pelgrom's scaling law [12] is used to model the area dependency of $\sigma_{\Delta V_{T H}}, \sigma_{\Delta \beta / \beta}$ and $\sigma_{\Delta S S / S S}$ :

$$
\sigma_{\Delta V_{T H}}=\frac{A_{V T}}{\sqrt{W L}} \sigma_{\Delta \beta / \beta}=\frac{A_{\beta}}{\sqrt{W L}} \sigma_{\Delta S S / S S}=\frac{A_{S S}}{\sqrt{W L}}
$$

where $A_{V T}, A_{\beta}$ and $A_{S S}$ are the threshold-voltage, currentfactor and subthreshold-swing area-scaling parameters, respectively, and $W L$ is the device active area. By combining Eq. (8) with (5) and (7), the drain-current mismatch for any device geometry can be computed.

Finally, it is important to note that the model parameters in Eq. (5) and (7) $\left(\sigma_{\Delta V_{T H}}, \sigma_{\Delta \beta / \beta}\right.$ and $\left.\sigma_{\Delta S S / S S}\right)$ have not been selected to fit the proposed models to the measured data. Instead, the mismatch in each parameter $\left(V_{T H}, \beta, S S\right)$ was extracted from the $\mathrm{I}_{\mathrm{D}}-\mathrm{V}_{\mathrm{G}}$ data of each pair and subsequently used to compute the respective standard deviation used in the models described by Eq. (5) and (7). This is a strong argument for the validity of the adopted model.

\section{EXPERIMENTAL RESULTS}

Fig. 5 shows the $I_{D}-V_{G}$ characteristics for 48 NMOS and PMOS devices from a single die at RT, $100 \mathrm{~K}$ and LHT. The temperature impact on the three relevant parameters can clearly be identified: $V_{T H}$ increases $\left(V_{T H_{4.2 K}}-V_{T H_{300 K}} \approx\right.$ $100 \mathrm{mV})$, mobility increases $\left(\beta_{4.2 K} / \beta_{300 K} \approx 2 \times\right)$ and $S S$ decreases $\left(S S_{300 K} \approx 90 \mathrm{mV} / \mathrm{dec} \rightarrow S S_{4.2 K} \approx 20 \mathrm{mV} / \mathrm{dec}\right)$, which is consistent with prior works [4], [6], [22].

To investigate the cryogenic subthreshold drain-current behavior, additional measurements were carried out over a larger set of temperatures. Fig. 6a shows the $I_{D}-V_{G}$ curves of a single $\mathrm{W} / \mathrm{L}=1.2 \mu / 40 \mathrm{n}$ NMOS device at intermediate temperatures between RT and LHT in steps of $40 \mathrm{~K}$. It is clear from this plot that the $S S$ value decreases down to $40 \mathrm{~K}$ after which it starts to saturate.

To study the impact of device geometry on SS, Fig. $6 \mathrm{~b}$ shows data at LHT for NMOS devices from all available geometries, demonstrating a very weak sensitivity to device geometry. 

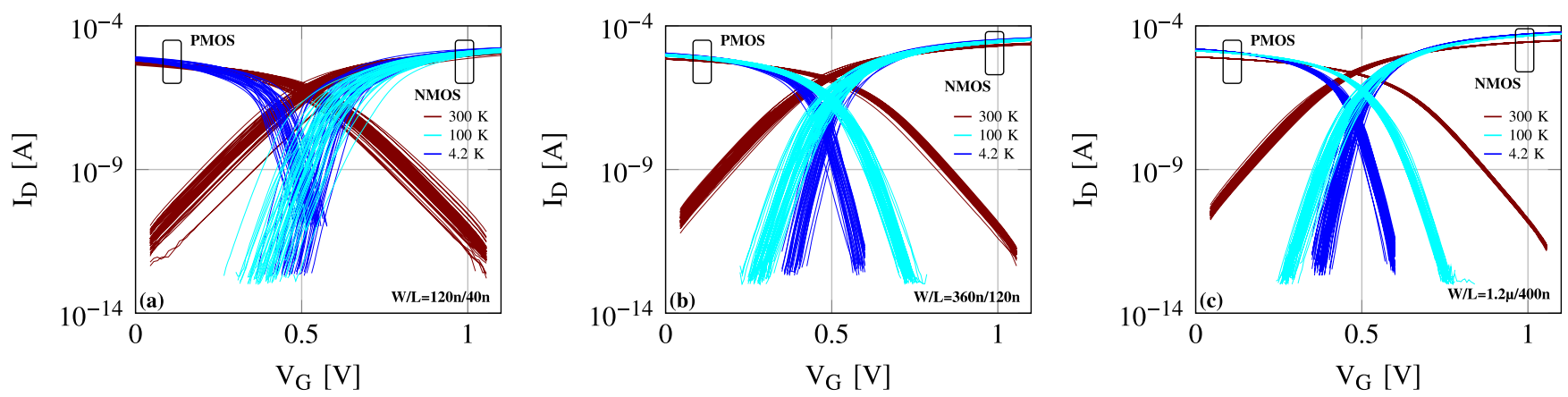

FIGURE 5. $I_{D}-V_{G}$ curves for a single die (48 devices per geometry) at $T=300 \mathrm{~K}, 100 \mathrm{~K}$ and $4.2 \mathrm{~K}$ : (a) $\mathrm{W} / \mathrm{L}=120 \mathrm{n} / 40 \mathrm{n}$; (b) $\mathrm{W} / \mathrm{L}=360 \mathrm{n} / 120 \mathrm{n}$ and (c) $W / L=1.2 \mu / 400 n$. $\left|V_{D S}\right|=50 \mathrm{mV}$. $V_{S}=0 \mathrm{~V}$ for NMOS and $1.1 \mathrm{~V}$ for PMOS, respectively.
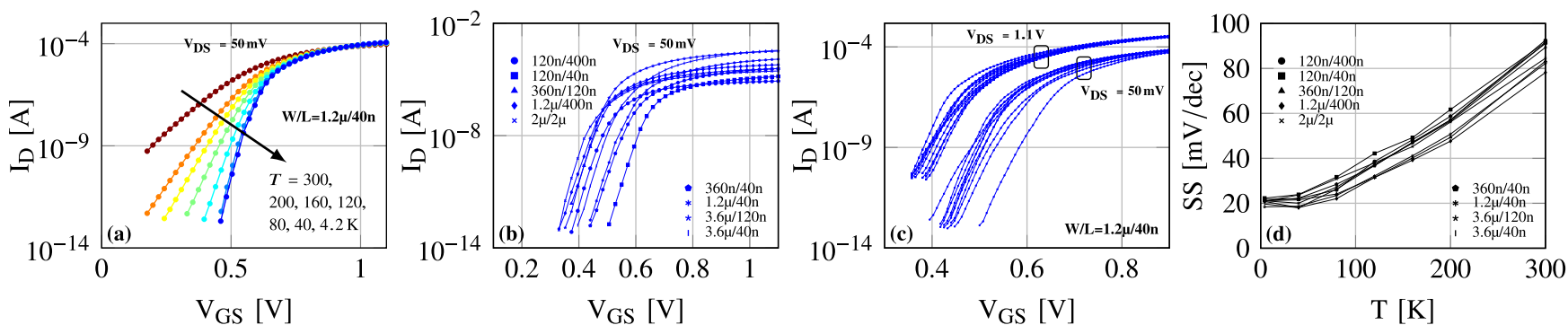

FIGURE 6. NMOS device behaviour over temperature: (a) $I_{D}-V_{G}$ curves of a $W / L=1.2 \mu / 40$ n device; (b) $I_{D}-V_{G}$ curves of all 9 geometries at $T=4.2 \mathrm{~K}$; (c) $I_{D}-V_{G}$ curves of $10 \mathrm{~W} / L=1.2 \mu / 40$ devices at $T=4.2 \mathrm{~K}$ and $(\mathrm{d})$ extracted $S S\left(V_{D S}=50 \mathrm{mV}\right)$ as function of temperature for all 9 geometries.
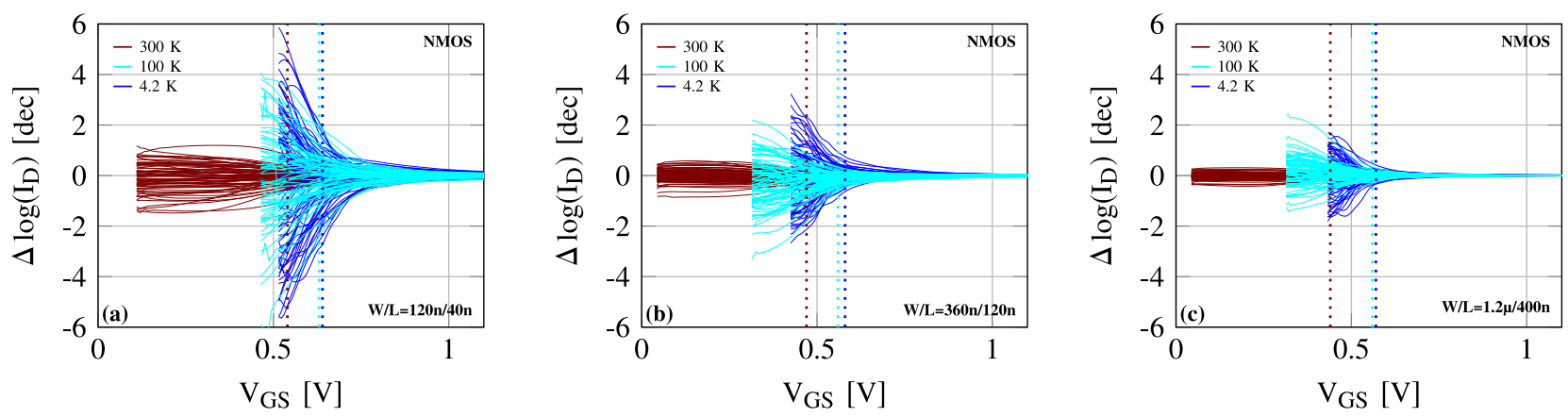

FIGURE 7. NMOS drain-current mismatch of 72 device pairs ( 3 dies) at $T=300 \mathrm{~K}, 100 \mathrm{~K}$ and $4.2 \mathrm{~K}$ : (a) $\mathrm{W} / \mathrm{L}=120 \mathrm{n} / 40 \mathrm{n}$; (b) $\mathrm{W} / \mathrm{L}=360 \mathrm{n} / 120 \mathrm{n}$ and (c) $\mathrm{W} / \mathrm{L}=1.2 \mu / 400 \mathrm{n}$. $V_{T H}$ marked by dashed lines. $V_{D S}=50 \mathrm{mV}$.

To assess the subthreshold drain-current variability for devices with equal geometry, the drain current of $10 \mathrm{NMOS}$ devices with $\mathrm{W} / \mathrm{L}=1.2 \mu / 40 \mathrm{n}$ at $\mathrm{T}=4.2 \mathrm{~K}$, biased both in triode $\left(V_{D S}=50 \mathrm{mV}\right)$ and saturation $\left(V_{D S}=1.1 \mathrm{~V}\right)$, is reported in Fig. 6c. A slight $V_{T H}$ decrease due to DIBL and a subthreshold current increase can clearly be seen for the saturated devices with respect to those operated in triode. The impact of the $V_{T H}$ and $S S$ variability on the subthreshold drain-current distribution is clearly visible in this plot.

To be complete, the temperature behaviour of the extracted $S S$ for all 9 available NMOS geometries is shown in Fig. 6d. $S S$ improves with decreasing temperature up to $4 \times$ from RT to LHT. At temperatures below $40 \mathrm{~K}$, the slope saturates (see Fig. 6a). Similar curves are obtained for the PMOS devices.

To investigate subthreshold mismatch, $\Delta \log \left(I_{D}\right)=$ $\log \left(I_{D 1}\right)-\log \left(I_{D 2}\right)$ was computed for 72 device pairs at
RT, $100 \mathrm{~K}$ and LHT, as plotted in Fig. 7. The combination of the increased $V_{T H}$ and the steeper $S S$ causes $I_{D}$ to reach the instrument's current floor at a much higher $V_{G S}$ at cryogenic temperatures compared to RT. The subthreshold mismatch is orders of magnitude higher at LHT compared to RT, which is attributed to an increased $S S$ mismatch further exacerbated by the steeper subthreshold slope.

Pelgrom plots for $\sigma_{\Delta V_{T H}}, \sigma_{\Delta \beta / \beta}$ and $\sigma_{\Delta S S / S S}$ are shown in Fig. 8 and Fig. 9 for PMOS and NMOS, respectively. The linear fittings are inversely weighted with the $95 \%$ confidence bounds, with the slopes representing the A-factors in Eq. (8). For both NMOS and PMOS devices, the Pelgrom law is effective in modeling $\sigma_{\Delta V_{T H}}$ and $\sigma_{\Delta \beta / \beta}$ at RT, $100 \mathrm{~K}$ and LHT.

The $\sigma_{\Delta S S / S S}$ dependency on area plotted in Fig. 8c and Fig. $9 \mathrm{c}$ can be successfully modeled by the Pelgrom law at 

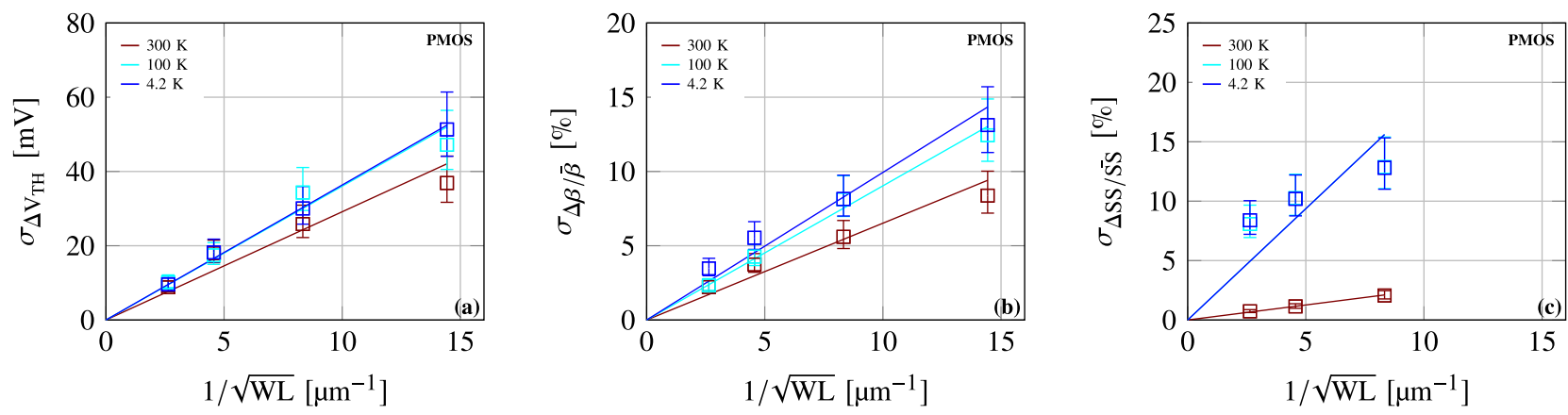

FIGURE 8. Pelgrom plots of PMOS with $L=40$ n device length at $T=300 \mathrm{~K}, 100 \mathrm{~K}$ and $4.2 \mathrm{~K}$ for: (a) $\sigma_{\Delta V_{T H}}$ (b) $\sigma_{\Delta \beta / \beta}$ and (c) $\sigma_{\Delta S S / S S}$. 95\% confidence intervals are shown.
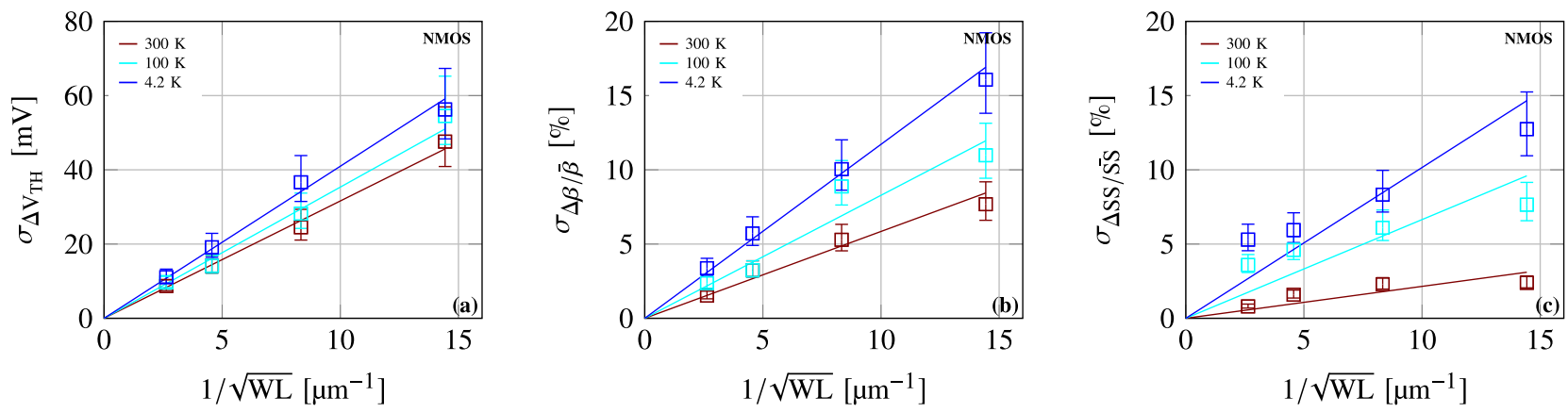

FIGURE 9. Pelgrom plots of NMOS with L=40n device length at $\mathrm{T}=300 \mathrm{~K}, 100 \mathrm{~K}$ and $4.2 \mathrm{~K}$ for: (a) $\sigma_{\Delta V_{T H}}$ (b) $\sigma_{\Delta \beta / \beta}$ and (c) $\sigma_{\Delta S S / S S}$. 95\% confidence intervals are shown.
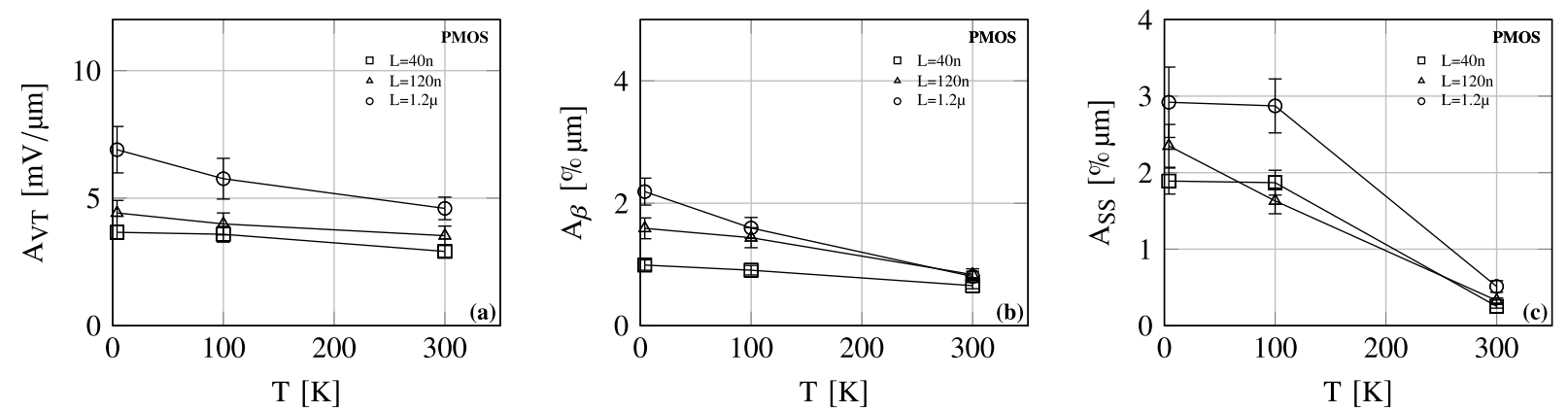

FIGURE 10. A-factors as a function of temperature for 3 different PMOS device lengths: (a) $A_{V T}$; (b) $A_{\beta}$ and (c) $A_{S S} .95 \%$ confidence intervals are shown.
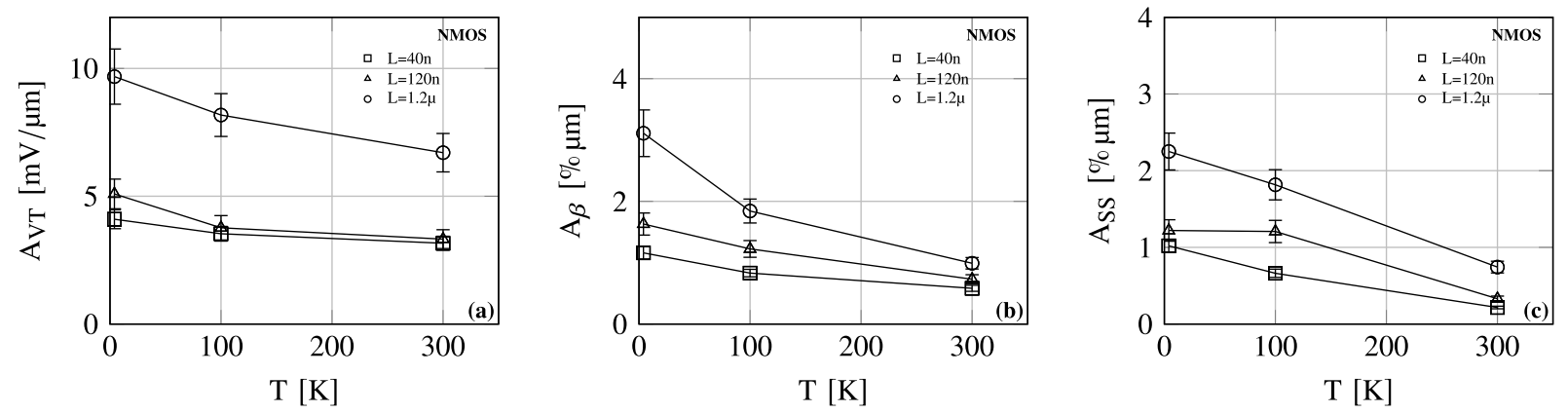

FIGURE 11. A-factors as a function of temperature for 3 different device NMOS lengths: (a) $A_{V T}$; (b) $A_{\beta}$ and (c) $A_{S S}$. 95\% confidence intervals are shown.

RT, $100 \mathrm{~K}$ and LHT. Only devices with $\mathrm{L}=40 \mathrm{n}$ are shown in these plots for clarity.

The temperature and length dependency of the extracted A-factors is shown in Fig. 10 and Fig. 11 with error bars representing $95 \%$ confidence intervals. For both NMOS and PMOS, $A_{V T}$ shows a significant increase of $\sim 1.5 \times$ at LHT with respect to $\mathrm{RT}$ for $\mathrm{L}=400 \mathrm{n}$, while no significant increase is observed for $\mathrm{L}=40 \mathrm{n}$. $A_{\beta}$ increases significantly over the 

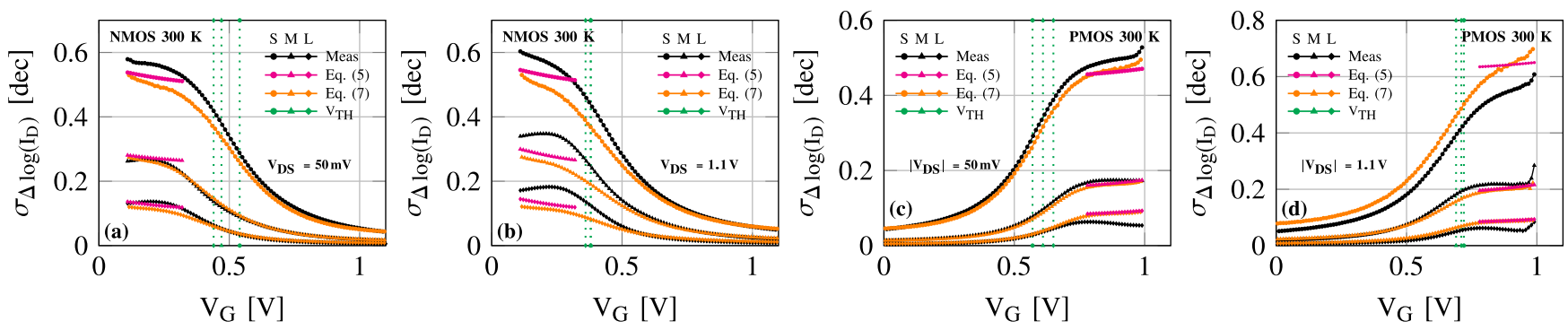

FIGURE 12. Drain-current mismatch as function of gate bias for 3 geometries at $T=300 \mathrm{~K}: S(W / L=120 \mathrm{n} / 40 \mathrm{n})$; $M(W / L=360 \mathrm{n} / 120 \mathrm{n})$ and $\mathrm{L}$ $(\mathrm{W} / \mathrm{L}=1.2 \mu / 400 \mathrm{n}) . V_{T H}$ and $V_{D S}$ indicated in each figure. $V_{S}=0 \mathrm{~V}$ for NMOS and $1.1 \mathrm{~V}$ for PMOS.
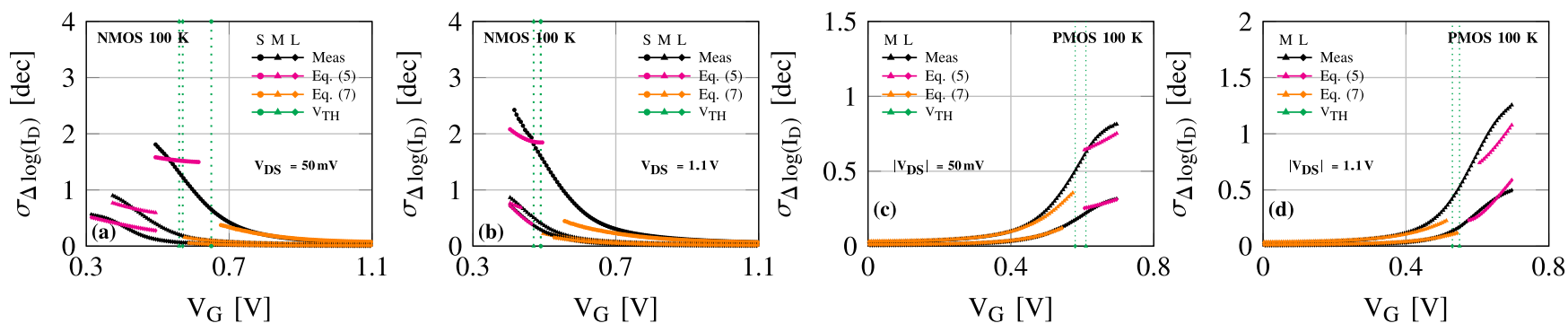

FIGURE 13. Drain-current mismatch as function of gate bias for 3 geometries at $T=100 \mathrm{~K}: S(W / L=120 \mathrm{n} / 40 \mathrm{n}) ; M(W / L=360 \mathrm{n} / 120 \mathrm{n})$ and $L$ $(\mathrm{W} / \mathrm{L}=1.2 \mu / 400 \mathrm{n}) . V_{T H}$ and $V_{D S}$ indicated in each figure. $V_{S}=0 \mathrm{~V}$ for NMOS and $1.1 \mathrm{~V}$ for PMOS.
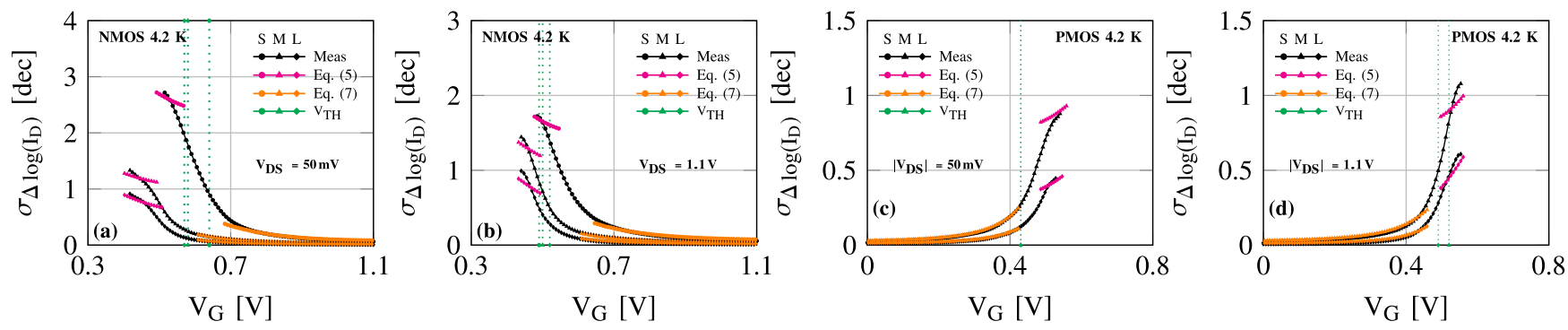

FIGURE 14. Drain-current mismatch as function of gate bias for 3 geometries at $T=4.2 \mathrm{~K}: \mathrm{S}(\mathrm{W} / \mathrm{L}=120 \mathrm{n} / 40 \mathrm{n}) ; \mathrm{M}(\mathrm{W} / \mathrm{L}=360 \mathrm{n} / 120 \mathrm{n})$ and $\mathrm{L}$ $(\mathrm{W} / \mathrm{L}=1.2 \mu / 400 \mathrm{n})$. $\mathrm{V}_{T H}$ and $\left|\mathrm{V}_{D S}\right|$ indicated in each figure. $\mathrm{V}_{S}=0 \mathrm{~V}$ for NMOS and $1.1 \mathrm{~V}$ for PMOS.

same temperature range: $\sim 2 \times$ for $\mathrm{N}-$ and PMOS devices with $\mathrm{L}=40 \mathrm{n}$ and up to $\sim 4.2 \times$ for $\mathrm{L}=400 \mathrm{n}$ NMOS devices, respectively. For all lengths, $A_{S S}$ significantly increases by $\sim 3 \times$ and $\sim 5 \times$ at $100 \mathrm{~K}$ compared to RT for NMOS and PMOS devices, respectively. Below $100 \mathrm{~K}, A_{S S}$ saturates or increases only slightly.

Drain-current mismatch for devices operating in triode and saturation at various temperatures and geometries is plotted in Fig. 12, 13 and 14, together with the Croon and the subthreshold models of Eq. (5) and (7). It should be noted that the $\sigma_{\Delta V_{T H}}, \sigma_{\Delta \beta / \beta}$ and $\sigma_{\Delta S S / S S}$ used in these two equations are the same as reported for the Pelgrom plots and have been directly derived from the measurements and not by fitting the models. Only 3 of the 9 available geometries are shown here for brevity, but similar trends hold for the omitted curves.

At $300 \mathrm{~K}$, in Fig. 12, both the Croon and the subthreshold models are able to predict mismatch over the full bias range for both NMOS and PMOS and in all operating regimes. At RT, drain-current mismatch reaches a plateau in the subthreshold region due to the low variability of $S S$ at RT.
Drain-current mismatch at $100 \mathrm{~K}$, as plotted in Fig. 13, shows an increased subthreshold mismatch compared to RT. The Croon model is capable of fitting the data in strong to moderate inversion, but it ceases to be adequate in subthreshold. The subthreshold model is able to give a better prediction in this region.

In Fig. 14, the subthreshold mismatch at LHT increases further compared to $100 \mathrm{~K}$, which is in line with the $\Delta \log \left(I_{D}\right)$ plots in Fig. 7 while the trend of the curves stays similar with those at RT and $100 \mathrm{~K}$. The Croon model is again able to predict mismatch in strong to moderate inversion but the subthreshold model needs to be employed for weak inversion.

\section{DISCUSSION}

As mentioned in Section II-A, the anomalous behavior previously reported in [25] was identified as an artifact due to an excessive parasitic resistance in series to the gate of the DUT. Although those artifacts have been eliminated in this paper, discontinuities in the $S S$ were still observed at 

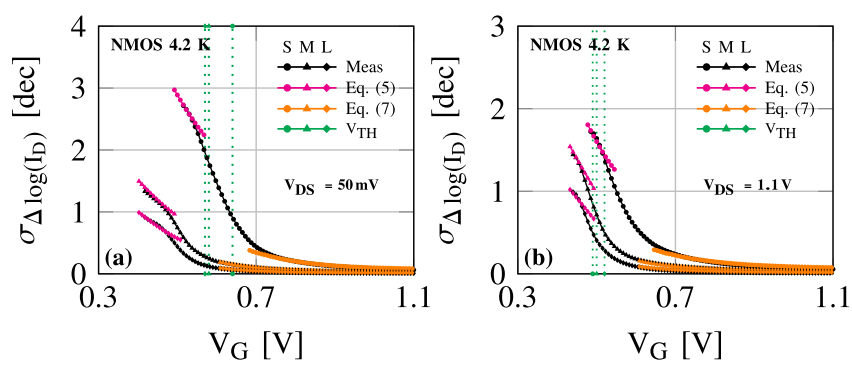

FIGURE 15. Drain-current mismatch modeled using the Croon and the subthreshold model and tuning $\sigma_{\Delta S S / S S}$ and $\sigma_{\Delta} V_{T H}$ for the best fit between experimental data and models: (a) $V_{D S}=50 \mathrm{mV}$; (b) $V_{D S}=1.1 \mathrm{~V}$.

$4.2 \mathrm{~K}$ both in the multiplexed devices and in separate bare, pad-accessible devices, similar to the observations in prior work [7], [29]-[32]. Such behavior was attributed to resonant electron/hole tunneling through a quantum dot (QD) [29] or through the electronic states of dopants [7], [31], [32], to the freeze-out of superficial impurities [30] or to conduction at the device edges [30]. Because of the significant random nature of those mechanisms, a severe impact on subthreshold matching could be expected. However, since the $S S$ is extracted here from the average subthreshold slope over a wide current range, local discontinuities of SS cannot be captured. Although the model in Fig. 14 uses the variability of the $S S$ extracted in this way, it is still able to accurately match the measured data, thus pointing to a negligible impact of those small SS discontinuities on the subthreshold mismatch statistics.

The observed length dependency of $\sigma_{\Delta V_{T H}}$ and $\sigma_{\Delta \beta / \beta}$ (see Fig. 8 and 9) is compatible with prior results presented in [22].

Based on the A-factors for $\mathrm{L}=40 \mathrm{n}$, for which the largest number of geometries are available and, hence the highest accuracy is reached, it can be concluded that $A_{V T}$ has no statistically significant variation with temperature, while $A_{\beta}$ and $A_{S S}$ increase significantly from RT to LHT.

As highlighted in Section IV, the Croon model is able to accurately predict mismatch in moderate to strong inversion at all temperatures and bias conditions, as also demonstrated in [22], while the subthreshold model must be adopted in weak inversion. Although the models and the experimental data already show good agreement when using $\sigma_{\Delta V_{T H}}$, $\sigma_{\Delta \beta / \beta}$ and $\sigma_{\Delta S S / S S}$ extracted from the measurements, as described in Section II-B and IV, an even smaller discrepancy is obtained when fitting the models described in Eq. (5) and (7) with the experimental data, as shown in Fig. 15.

\section{CONCLUSION}

This paper reports, for the first time, the characterization and modeling of the subthreshold device mismatch of CMOS devices at cryogenic temperatures. Mismatch increases at cryogenic temperatures in all operating regions and, in particular, subthreshold matching deteriorates by more than several orders of magnitude. As the Croon model does not take subthreshold-swing variability into account, a specific subthreshold model is shown to adequately model mismatch in the weak inversion region at these temperatures. Improving matching by increasing the device area as predicted by Pelgrom law proves to be valid at cryogenic temperatures in all operating regions including subthreshold. As a result, the proposed mismatch models can become an essential tool for the design of the cryo-electronics that will enable scalable quantum computers.

\section{ACKNOWLEDGMENT}

The authors would like to thank Dr. M. Veldhorst, Dr. H. P. Tuinhout, Dr. M. J. M. Pelgrom and G. Kiene for the helpful discussions.

\section{REFERENCES}

[1] L. Vandersypen, "Quantum computing - The next challenge in circuit and system design," in Proc. IEEE Int. Solid-State Circuits Conf. (ISSCC), Feb. 2017, pp. 24-29.

[2] F. Arute et al., "Quantum supremacy using a programmable superconducting processor," Nature, vol. 574, pp. 505-510, Oct. 2019.

[3] F. Sebastiano et al., "Cryo-CMOS electronic control for scalable quantum computing," in Proc. ACM/EDAC/IEEE Design Autom. Conf. (DAC), 2017, pp. 1-6.

[4] R. M. Incandela, L. Song, H. Homulle, E. Charbon, A. Vladimirescu, and F. Sebastiano, "Characterization and compact modeling of nanometer CMOS transistors at deep-cryogenic temperatures," IEEE J. Electron Devices Soc., vol. 6, pp. 996-1006, 2008.

[5] S. R. Ekanayake, T. Lehmann, A. S. Dzurak, R. G. Clark, and A. Brawley, "Characterization of SOS-CMOS FETs at low temperatures for the design of integrated circuits for quantum bit control and readout," IEEE Trans. Electron Devices, vol. 57, no. 2, pp. 539-547, Feb. 2010.

[6] A. Beckers, F. Jazaeri, and C. Enz, "Characterization and modeling of $28 \mathrm{~nm}$ bulk CMOS technology down to $4.2 \mathrm{~K}$," IEEE J. Electron Devices Soc., vol. 6, pp. 1007-1018, 2018.

[7] A. Beckers, F. Jazaeri, H. Bohuslavskyi, L. Hutin, S. D. Franceschi, and C. Enz, "Characterization and modeling of 28-nm FDSOI CMOS technology down to cryogenic temperatures," Solid-State Electron., vol. 159, pp. 106-115, Sep. 2019.

[8] A. Beckers, F. Jazaeri, and C. Enz, "Cryogenic MOS transistor model," IEEE Trans. Electron Devices, vol. 65, no. 9, pp. 3617-3625, Sep. 2018.

[9] S. Mennillo, A. Spessot, L. Vendrame, and L. Bortesi, "An analysis of temperature impact on MOSFET mismatch," in Proc. IEEE Int. Conf. Microelectron. Test Struct., Mar. 2009, pp. 56-61.

[10] J. J.-Y. Kuo, W. P.-N. Chen, and P. Su, "Temperature dependence of device mismatch and harmonic distortion in nanoscale uniaxialstrained pMOSFETs," in Proc. IEEE Int. Conf. IC Design Technol., May 2011, pp. 1-4.

[11] P. Andricciola and H. P. Tuinhout, "The temperature dependence of mismatch in deep-submicrometer bulk MOSFETs," IEEE Electron Device Lett., vol. 30, no. 6, pp. 690-692, Jun. 2009.

[12] M. J. M. Pelgrom, A. C. J. Duinmaijer, and A. P. G. Welbers, "Matching properties of MOS transistors," in Proc. ESSCIRC, Sep. 1988, pp. 327-330.

[13] J. A. Croon, W. M. C. Sansen, and H. Maes, Matching Properties of Deep Sub-Micron MOS Transistors. New York, NY, USA: Springer, 2005.

[14] J. J.-Y. Kuo, W. P.-N. Chen, and P. Su, "Investigation and analysis of mismatching properties for nanoscale strained MOSFETs," IEEE Trans. Nanotechnol., vol. 9, no. 2, pp. 248-253, Apr. 2010.

[15] K. Sakakibara and K. Arimoto, "An accurate method for predicting temperature-dependent current mismatch in meak inversion region of long and wide channel MOSFET without subthreshold hump," Jpn. J. Appl. Phys., vol. 53, no. 6, May 2014, Art. no. 064305.

[16] A. Pavasović, A. Andreou, and C. Westgate, "Characterization of subthreshold MOS mismatch in transistors for VLSI systems," J. VLSI Signal Process., vol. 6, pp. 75-85, Jul. 1994. 
[17] P. Magnone, F. Crupi, P. Andricciola, H. Tuinhout, and R. J. P. Lander, "FinFET mismatch in subthreshold region: Theory and experiments," IEEE Trans. Electron Devices, vol. 57, no. 11, pp. 2848-2856, Nov. 2010

[18] X. E. Bee, M. M. B. M. Fauzi, and P. B. Y. Tan, "Modeling of MOSFET subthreshold swing mismatch with BSIM4 model," in Proc. IEEE Int. Conf. Semicond. Electron. (ICSE), Aug. 2016, pp. 86-88.

[19] M. Denison, A. Pergoot, and M. Tack, "Prediction of MOS matching in weak and moderate inversion from threshold matching in strong inversion," in Proc. 28th Eur. Solid-State Device Res. Conf., Sep. 1998, pp. 648-651.

[20] N. Wils, H. Tuinhout, and M. Meijer, "Influence of STI stress on drain current matching in advanced CMOS," in Proc. IEEE Int. Conf. Microelectron. Test Struct., Mar. 2008, pp. 238-243.

[21] P. A. 't Hart, J. P. G. van Dijk, M. Babaie, E. Charbon, A. Vladimircscu, and F. Sebastiano, "Characterization and model validation of mismatch in nanometer CMOS at cryogenic temperatures," in Proc. 48th Eur. Solid-State Device Res. Conf. (ESSDERC), Sep. 2018, pp. 246-249.

[22] P. A. 't Hart, M. Babaie, E. Charbon, A. Vladimirescu, and F. Sebastiano, "Characterization and modeling of mismatch in cryoCMOS," IEEE J. Electron Devices Soc., vol. 8, pp. 263-273, 2020.

[23] K. Das and T. Lehmann, "Effect of deep cryogenic temperature on silicon-on-insulator CMOS mismatch: A circuit designer's perspective," Cryogenics, vol. 62, pp. 84-93, Jul./Aug. 2014.

[24] N. C. Dao, A. E. Kass, C. T. Jin, and P. H. W. Leong, "Impact of series resistance on bulk CMOS current matching over the 5-300K temperature range," IEEE Electron Device Lett., vol. 38, no. 7, pp. 847-850, Jul. 2017.

[25] P. A. 't Hart, M. Babaie, E. Charbon, A. Vladimirescu, and F. Sebastiano, "Subthreshold mismatch in nanometer CMOS at cryogenic temperatures," in Proc. 49th Eur. Solid-State Device Res. Conf. (ESSDERC), Sep. 2019, pp. 98-101.

[26] J. A. Croon, M. Rosmeulen, S. Decoutere, W. Sansen, and H. E. Maes, "An easy-to-use mismatch model for the MOS transistor," IEEE $J$. Solid-State Circuits, vol. 37, no. 8, pp. 1056-1064, Aug. 2002.

[27] A. Ortiz-Conde, F. G. Sánchez, J. J. Liou, A. Cerdeira, M. Estrada, and Y. Yue, "A Review of Recent MOSFET Threshold Voltage Extraction Methods," Microelectron. Rel., vol. 42, nos. 4-5, pp. 583-596, 2002.

[28] M. F. Hamer, "First-order parameter extraction on enhancement silicon MOS transistors," IEE Proc. I, Solid-State Electron Devices, vol. 133, no. 2, pp. 49-54, Apr. 1986.

[29] S. Bonen et al., "Cryogenic characterization of 22-nm FDSOI CMOS technology for quantum computing ICs," IEEE Electron Device Lett., vol. 40, no. 1, pp. 127-130, Jan. 2019.

[30] T. Elewa et al., "Performance and physical mechanisms in SIMOX MOS transistors operated at very low temperature," IEEE Trans. Electron Devices, vol. 37, no. 4, pp. 1007-1019, Apr. 1990.

[31] H. Bohuslavskyi et al., "28nm fully-depleted SOI technology: Cryogenic control electronics for quantum computing," in Proc. Silicon Nanoelectron. Workshop (SNW), Jun. 2017, pp. 143-144.

[32] R. Wacquez et al., "Single dopant impact on electrical characteristics of SOI NMOSFETs with effective length down to 10nm," in Proc. Symp. VLSI Technol., Jun. 2010, pp. 193-194.

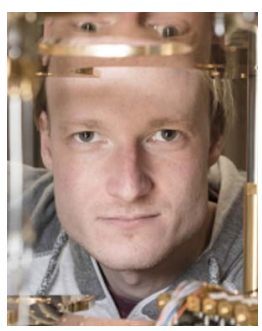

P. A. 'T HART received the M.Sc. degree in electrical engineering from the Delft University of Technology, The Netherlands, in 2014. He is currently pursuing the $\mathrm{Ph} . \mathrm{D}$. degree in characterizing and modeling cryogenic MOSFETs with the Group of Edoardo Charbon. He worked with the Electronic Components, Materials and Technology Department and the Electronic Instrumentation Laboratory, for his thesis, where he did characterization of the piezojunction effect in bipolar transistors. His research interests include low temperature electronics, modeling, and characterization.

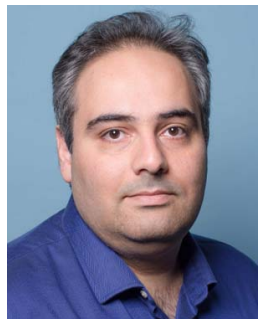

M. BABAE (Member, IEEE) received the Ph.D. degree (cum laude) in electrical engineering from the Delft University of Technology, Delft, The Netherlands, in 2016. In 2006, he joined the Kavoshcom Research and Development Group, Tehran, where he was involved in designing wireless communication systems. From 2009 to 2011, he was a CTO with Kavoshcom Research and Development Group. From 2014 to 2015, he was a Visiting Scholar Researcher with the Berkeley Wireless Research Center, Berkeley, CA, USA. In 2016, he joined the Delft University of Technology, where he is currently an Assistant Professor (Tenured). His current research interests include $\mathrm{RF}$ /millimeter-wave integrated circuits and systems for wireless communications, and cryogenic electronics for quantum computation. $\mathrm{He}$ was a co-recipient of the IEEE Solid-State Circuits Society Pre-Doctoral Achievement Award from 2015 to 2016, the IEEE ISSCC Best Demo Award in 2019, and the Veni Award from the Netherlands Organization for Scientific Research in 2019. He has been a Committee Member of the Student Research Preview of the IEEE International Solid-State Circuits Conference since 2017. He is currently serving on the Technical Program Committee of the IEEE European Solid-State Circuits Conference.

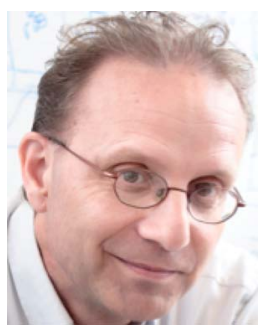

EDOARDO CHARBON (Fellow, IEEE) received the Diploma degree in electrical engineering and EECS from ETH Zurich, in 1988, the M.S. degree in electrical engineering and EECS from the University of California at San Diego in 1991, and the Ph.D. degree in electrical engineering and EECS from the University of California at Berkeley in 1995. He has consulted with numerous organizations, including Bosch, X-Fab, Texas Instruments, Maxim, Sony, Agilent, and Carlyle Group. He was with Cadence Design Systems from 1995 to 2000, where he was the Architect of the company's initiative on information hiding for intellectual property protection. In 2000, he joined Canesta Inc., as the Chief Architect, where he led the development of wireless 3-D CMOS image sensors. Since 2002, he has been a member of the faculty of EPFL, where he has been a Full Professor since 2015. From 2008 to 2016, he was with the Delft University of Technology's as a Chair of VLSI Design. He has been the driving force behind the creation of deepsubmicron CMOS SPAD technology, which is mass-produced since 2015 and is present in telemeters, proximity sensors, and medical diagnostics tools. He has authored or coauthored over 350 papers and two books, and holds 21 patents. His interests span from 3-D vision, FLIM, FCS, NIROT to super-resolution microscopy, time-resolved Raman spectroscopy, and cryoCMOS circuits and systems for quantum computing. He is a Distinguished Visiting Scholar with the W. M. Keck Institute for Space, Caltech and a Distinguished Lecturer with IEEE Photonics Society. He is a fellow of the Kavli Institute of Nanoscience Delft. 


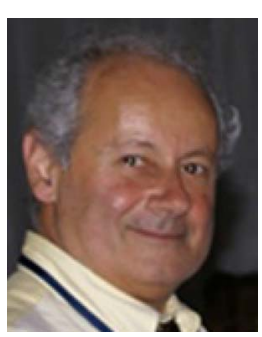

ANDREI VLADIMIRESCU (Fellow, IEEE) received the M.S. and Ph.D. degrees in EECS from the University of California at Berkeley, Berkeley, CA, USA, where he was a key contributor to the SPICE simulator, releasing the SPICE2G6 production-level SW in 1981. $\mathrm{He}$ pioneered electrical simulation on parallel computers with the CLASSIE simulator as part of his Ph.D. He has authored The SPICE Book (Wiley). He was a Research and Development Director leading the design and implementation of innovative software and hardware Electronic Design Automation products for Analog Devices Inc., Daisy Systems, Analog Design Tools, and Valid Logic and Cadence for many years. He is currently a Professor involved in research projects with the University of California at Berkeley, Delft University of Technology, and the Institut Supérieur d'Électronique de Paris, as well as consultant to industry. His research activities are in the areas of ultra-low-voltage CMOS, design, simulation, and modeling of circuits with new devices and circuits for quantum computing.

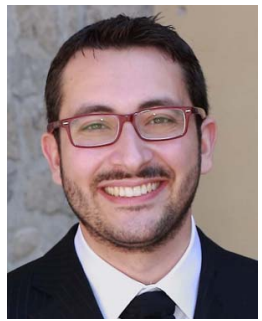

FABIO SEBASTIANO (Senior Member, IEEE) received the B.Sc. (cum laude) and M.Sc. (cum laude) degrees in electrical engineering from the University of Pisa, Italy, in 2003 and 2005, respectively, the M.Sc. degree (cum laude) from the Sant'Anna School of Advanced Studies, Pisa, Italy, in 2006, and the Ph.D. degree from the Delft University of Technology, The Netherlands, in 2011. From 2006 to 2013, he was with NXP Semiconductors Research, Eindhoven, The Netherlands, where he conducted research on fully integrated CMOS frequency references, deep-submicron temperature sensors, and area-efficient interfaces for magnetic sensors. In 2013, he joined Delft University of Technology, where he is currently an Assistant Professor. He has authored or coauthored one book, 11 patents, and over 60 technical publications. His main research interests are cryogenic electronics for quantum computing, quantum computing, sensor read-outs, and fully integrated frequency references. He was a co-recipient of the Best Student Paper at ISCAS in 2008, the Best Paper Award at IWASI in 2017, and the Best IP Award at DATE in 2018. He is an Associate Editor of the IEEE TRANSACTIONS ON VERY LARGE SCALE INTEgRATION (VLSI) SySTEMS. $\mathrm{He}$ is a Distinguished Lecturer of the Solid-State Circuit Society and a member of the Technical Program Committee of the RFIC Symposium. 